\title{
Event-based Data Scheduling for a Class of Interconnected Networked Control Systems
}

\author{
Mohammad H. Mamduhi, Frederik Deroo, and Sandra Hirche ${ }^{1}$
}

\begin{abstract}
In this paper we address the problem of eventbased data scheduling for a class of physically interconnected networked control systems which compete for limited communication resources. The overall interconnected system consists of multiple heterogeneous LTI sub-systems with the physical interconnection being modeled by a directed acyclic graph (DAG). The sub-systems are controlled by a networked controller through a shared communication channel. In order to cope with the limited channel capacity, we introduce a bi-character deterministic-probabilistic scheduling mechanism which dynamically assigns access priorities to each sub-system at each time-step according to an error-dependent priority measure. The sub-systems which are granted channel access then transmit their state information through the communication network. Given an emulation-based control strategy, we prove stability of such networked systems under the proposed scheduling law in terms of $f$-ergodicity of overall network-induced error. Simulation results illustrate the proposed approach and show a reduction in the error variance compared to standard TDMA and uniform random-access scheduling policies.
\end{abstract}

\section{INTRODUCTION}

Interconnected networked control systems represent a system class with application examples ranging from infrastructure systems, e.g. distributed electrical power, gas and water systems to mechatronic systems like large-scale telescopes. The unifying property is that several components are physically interconnected. Besides classical centralized control methods, a different approach is to use decentralized control, i.e. control based on local information [1]. As a compromise between centralized and decentralized control, distributed control methods have emerged that exploit partial information exchange among local controllers. Recently, many results have treated distributed control of interconnected systems, see e.g. [2], [3] among many others.

In general, all these results assume a continuous or timetriggered information exchange with periodic sampling. In practice, however, typically a shared communication medium is employed, and information exchange is subject to several complications, e.g. communication capacity limitations and congestion, time delay and packet dropouts. These problems can deteriorate the control performance, and even lead to instability. In order to make efficient use of limited communication resources, event-triggered control and scheduling strategies have been investigated in recent years [4]-[6].

These approaches entail that sampled values are transmitted when a certain event happens instead of transmitting

\footnotetext{
${ }^{1}$ M. H. Mamduhi, F. Deroo and S. Hirche are with the Chair of Information-oriented Control, Technische Universität München, Arcisstraße 21, D-80290 München, Germany; http: / / www.itr.ei.tum.de, \{mh.mamduhi, fred.deroo, hirche\}etum.de
}

at periodic time instants. Events are typically triggered by either deterministic or stochastic rules. A basic deterministic scheduling approach is the Try-Once-Discard (TOD) that awards the medium access to the system with the largest estimation error and discards the remaining requests [6]. Stability of such systems are studied by computing the Maximal Allowable Transfer Interval (MATI) which finds an upper bound on the interval between two successive transmissions [7]. Deterministic policies however are not well suited to deal with delays, dropouts, collisions and noisy systems [8]. Alternatively, probabilistic policies have been investigated that consider stochastic NCSs [9], [10]. These methods are better equipped to deal with noise and collisions but also with model uncertainties [11]-[13]. The aforementioned results deal with NCSs with isolated subsystems, i.e. without physical interconnection.

The main contribution of this paper is a novel scheduling scheme for an interconnected NCS. The system consists of multiple physically interconnected LTI sub-systems which are additionally coupled through a shared communication network. A scheduler collects the error signals from all subsystems to assign priorities and then awards the channel access based on a biased randomization. The presented approach guarantees stability of the interconnected NCS where the interconnections are represented by direct acyclic graphs (DAGs) and each node of the DAG has access to the information from all nodes affecting its dynamics either direct neighboring nodes or multi-hop neighbors. DAGs are used to model systems with some sort of hierarchy, e.g. vehicle platoons [14]. Exploiting an emulative control approach, we show stability of the resulting interconnected NCS in terms of $f$-ergodicity. Furthermore, the proposed approach promises better performance than standard timetriggered and idealized CSMA approaches.

As the remainder of this paper, the problem statement is described in Section II and follows by some preliminaries of Markov chains' stability. In Section III, we analyze the stability of such NCSs under the proposed scheduling law. Simulation results are presented in Section IV.

Notation. The Euclidean norm, conditional expectation, and Gaussian distribution with mean $\mu$ and covariance matrix $X$ are denoted $\|\cdot\|_{2}, \mathrm{E}[\cdot \cdot \cdot]$ and $\mathcal{N}(\mu, X)$, respectively. A state vector with superscript $i$ belongs to control loop $i$, while the subscript $k$ denotes the time-step. For matrices, subscript $i$ indicates the corresponding sub-system while superscript $n$ denotes the matrix power. A connected graph with set of vertices $\mathcal{V}$ and set of directed edges $\mathcal{E}$ is represented by $\mathcal{G}_{c}(\mathcal{V}, \mathcal{E})$. A node $j \in \mathcal{V}$ is an affecting node if at least 
one node $i$ exists between which a directed path from $j$ to $i$ is established. Node $i$ is called an affected node. If the path's length is one, node $j$ is a direct neighbor of node $i$. A node $l \in \mathcal{V}$ is called multi-hop neighbor of node $i$ if a directed path of length greater than one exists from $l$ to $i$.

\section{PROBLEM STATEMENT AND PRELIMINARIES}

Consider an NCS composed of $N$ heterogeneous LTI subsystems which are physically interconnected and are additionally coupled through a shared communication network, as depicted in Fig. 1. Each controlled sub-system consists of a stochastic discrete time LTI system $\mathcal{P}_{i}$ and a controller $\mathcal{C}_{i}$, and utilizes the communication network to transmit its state information. We assume that the transmitted data is accessible for all corresponding affected sub-systems. Therefore, control unit $\mathcal{C}_{i}$ corresponding to sub-system (node) $i$ has access to the transmitted information from the direct and multi-hop neighbors of node $i$. To allocate the capacitylimited channel among sub-systems, a scheduler decides whether the $i^{\text {th }}$ state $x_{k}^{i} \in \mathbb{R}^{n_{i}}$ is an event to be scheduled for channel access. A directed acyclic graph (DAG) $\mathcal{G}_{c}$ represents the physical interconnections between the subsystems $i \in\{1, \ldots, N\}$. An edge from node $j$ to node $i$ indicates that the dynamics of $i^{\text {th }}$ node is directly affected by $j^{\text {th }}$ one. We define the set of all direct or multi-hop neighbors of a node $i$ as $\tilde{S}_{n}^{i}$, while the set of only direct neighbors is denoted by $S_{n}^{i}$. The dynamics of a sub-system $\mathcal{P}_{i}$ then follows from the stochastic difference equation

$$
x_{k+1}^{i}=A_{i} x_{k}^{i}+B_{i} u_{k}^{i}+\sum_{j \in S_{n}^{i}} A_{i j} x_{k}^{j}+w_{k}^{i},
$$

where the noise sequence $w_{k}^{i} \in \mathbb{R}^{n_{i}}$ is i.i.d. with $\mathcal{N}(0, I)$ at each time-step $k$, and $A_{i} \in \mathbb{R}^{n_{i} \times n_{i}}, A_{i j} \in \mathbb{R}^{n_{i} \times n_{j}}, B_{i} \in$ $\mathbb{R}^{n_{i} \times m_{i}}$ describe system, interconnection and input matrices, respectively. The initial state $x_{0}^{i}$ is randomly chosen from an arbitrary distribution with bounded variance. Concatenation of the system state leads to the overall networked system

$$
x_{k+1}=A x_{k}+B u_{k}+w_{k},
$$

where $x=\left[x^{1^{\top}}, \ldots, x^{N^{\top}}\right]^{\top} \in \mathbb{R}^{n}, u=\left[u^{1^{\top}}, \ldots, u^{N^{\top}}\right]^{\top} \in$ $\mathbb{R}^{m}, A \in \mathbb{R}^{n \times n}$ consists of the blocks $A_{i}$ on the diagonal, and $A_{i j}$ on the off-diagonal, and $B \in \mathbb{R}^{n \times m}$ is block-diagonal with the entries $B_{i}$. Clearly, $\sum_{i=1}^{N} n_{i}=n$ and $\sum_{i=1}^{N} m_{i}=m$. The scheduler decides which sub-systems utilize the channel at each time-step $k$, by the binary variable $\delta_{k}^{i} \in\{0,1\}$ as

$$
\delta_{k}^{i}= \begin{cases}1, & x_{k}^{i} \text { sent through the channel } \\ 0, & x_{k}^{i} \text { blocked. }\end{cases}
$$

Following an emulation-based control approach we assume a stabilizing control law to be given for the system without any capacity constraints. Assume that the concatenated system (2) is driven by a state feedback control $u_{k}=\left[u_{k}^{1^{\top}}, \ldots, u_{k}^{N^{\top}}\right]^{\top}$. According to the information pattern, each node has access to the information about its own affecting nodes in the interconnection graph. The affecting nodes of a certain node $i$ include direct and multihop neighbors of node $i$. Thus, the control unit $\mathcal{C}_{i}$ can exploit

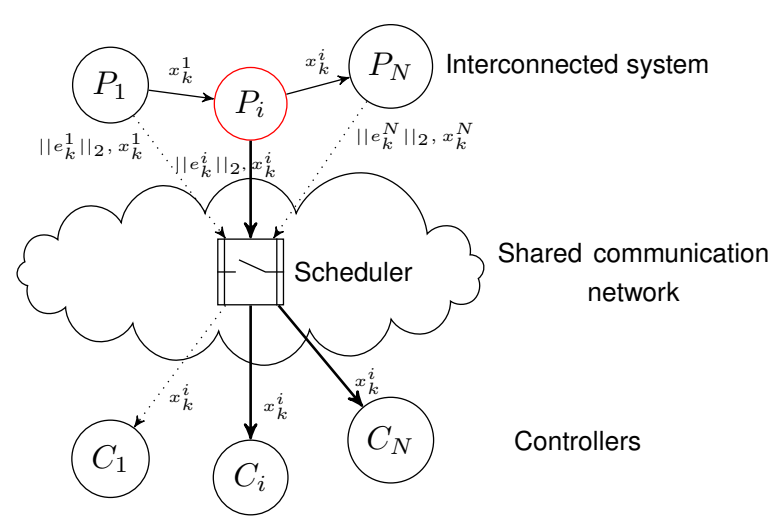

Fig. 1. A physically interconnected NCS over a shared communication channel with error-dependent centralized scheduler.

this available information to compute the control signal $u_{k}^{i}$. This leads to a distributed control law having the same DAG structure as the interconnection graph. The control signal $u_{k}^{i}$ is updated at every time-step $k$ either by the true state values $x_{k}^{i}$ from the local sub-system and $x_{k}^{j}$ from the affecting nodes $j \in \tilde{S}_{n}^{i}$, or by the estimated states $\hat{x}_{k}^{i}$ and $\hat{x}_{k}^{j}$ :

$$
u_{k}^{i}=-L_{i} \mathrm{E}\left[x_{k}^{i} \mid \mathcal{I}_{k}^{i}\right]-\sum_{j \in \tilde{S}_{n}^{i}} L_{i j} \mathrm{E}\left[x_{k}^{j} \mid \mathcal{I}_{k}^{i}\right],
$$

where $\mathcal{I}_{k}^{i}=\left\{z_{0}^{i}, \ldots, z_{k-1}^{i}\right\} \cup_{j \in \tilde{S}_{n}^{i}}\left\{z_{0}^{j}, \ldots, z_{k-1}^{j}\right\}$ is the set of information at node $i, L_{i}$ and $L_{i j}$ are feedback gains, and

$$
z_{k}^{i}= \begin{cases}x_{k}^{i}, & \delta_{k}^{i}=1 \\ \varnothing, & \delta_{k}^{i}=0 .\end{cases}
$$

Assuming stabilizability of the concatenated system (2), i.e. pair $(A, B)$ is stabilizable, an emulative control law, e.g. [3], ensures the gain $L$ consisting of blocks $L_{i}$ on the diagonal and $L_{i j}$ on the off-diagonal is stabilizing, i.e. the closed-loop matrix $(A-B L)$ is Hurwitz. In addition, a sub-system $i$ has knowledge of the matrices $A_{j}, B_{j}$ and feedback gains $L_{i j}$ from all nodes $j \in \tilde{S}_{n}^{i}$.

According to (3), $u_{k}^{i}$ is updated with true state values $x_{k}^{i}$ and $x_{k}^{j}$ if all direct neighbors $j \in S_{n}^{i}$ transmit at time-step $k$ along with the $i^{\text {th }}$ node itself. In case of a non-transmission from either the node $i$ or a direct neighboring node $j$, the estimates $\hat{x}_{k}^{i}$ or $\hat{x}_{k}^{j}$ are computed by a model-based estimator. The state estimate $\hat{x}_{k}^{j}$ of a node $j \in S_{n}^{i}$ at node $i$, i.e. $\mathrm{E}\left[x_{k}^{j} \mid \mathcal{I}_{k}^{i}\right]$ coincides with the estimation of $x_{k}^{j}$ computed at node $j$, i.e. $\mathrm{E}\left[x_{k}^{j} \mid \mathcal{I}_{k}^{j}\right]$. This follows from the fact that, according to the DAG interconnection structure, if $j \in S_{n}^{i}$, the information set $\mathcal{I}_{k}^{i}$ includes the information set $\mathcal{I}_{k}^{j}$, i.e. $\mathcal{I}_{k}^{j} \subset \mathcal{I}_{k}^{i}$. Knowing this, we have from (1) and (3):

$$
\begin{aligned}
\mathrm{E}\left[x_{k}^{i} \mid \mathcal{I}_{k}^{i}\right] & =\left(A_{i}-B_{i} L_{i}\right) \mathrm{E}\left[x_{k-1}^{i} \mid \mathcal{I}_{k-1}^{i}\right] \\
& +\sum_{j \in S_{n}^{i}}\left(A_{i j}-B_{i} L_{i j}\right) \mathrm{E}\left[x_{k-1}^{j} \mid \mathcal{I}_{k-1}^{j}\right],
\end{aligned}
$$

with initial distribution $\mathrm{E}\left[x_{0}^{i} \mid \mathcal{I}_{0}^{i}\right]=\mathrm{E}\left[x_{0}^{j} \mid \mathcal{I}_{0}^{j}\right]=0$. The network-induced error of a node $i$ at time-step $k$ is defined as the difference between actual and estimated state values:

$$
e_{k}^{i}=x_{k}^{i}-\mathrm{E}\left[x_{k}^{i} \mid \mathcal{I}_{k-1}^{i}\right] \text {. }
$$


Defining the aggregate network state $\left[x_{k}^{i^{\top}}, e_{k}^{i^{\top}}\right]^{\top}$ at node $i$, the dynamics of the $i^{\text {th }}$ node follows from (1)-(5):

$$
\begin{aligned}
{\left[\begin{array}{c}
x_{k+1}^{i} \\
e_{k+1}^{i}
\end{array}\right] } & =\left[\begin{array}{cc}
\left(A_{i}-B_{i} L_{i}\right) & \left(1-\delta_{k}^{i}\right) B_{i} L_{i} \\
0 & \left(1-\delta_{k}^{i}\right) A_{i}
\end{array}\right]\left[\begin{array}{c}
x_{k}^{i} \\
e_{k}^{i}
\end{array}\right]+\left[\begin{array}{l}
1 \\
1
\end{array}\right] w_{k}^{i} \\
& +\sum_{j \in S_{n}^{i}}\left[\begin{array}{cc}
\left(A_{i j}-B_{i} L_{i j}\right) & \left(1-\delta_{k}^{j}\right) B_{i} L_{i j} \\
0 & \left(1-\delta_{k}^{j}\right) A_{i j}
\end{array}\right]\left[\begin{array}{c}
x_{k}^{j} \\
e_{k}^{j}
\end{array}\right] .
\end{aligned}
$$

Remark 1: The control law in this paper can possess decentralized and distributed structures as long as it is stabilizing. For notational convenience and to achieve better performance, we assume a distributed control law with a structure that is identical to the physical interconnection DAG.

Note that, in accordance with the emulation-based framework, the control law synthesis is not explicitly addressed, i.e., design the feedback gains $L_{i}$ and $L_{i j}$. Instead, knowing the feedback gain $L$ stabilizes the system state (2) in the absence of capacity constraints, we focus on the scheduling design when communication resources are limited.

The aggregate network state (6) has triangular dynamics for each sub-system $i$ implying that the evolution of the local error state $e_{k}^{i}$ is independent of the local system state $x_{k}^{i}$ and neighboring states $x_{k}^{j}$. From (6), we have

$$
e_{k+1}^{i}=\left(1-\delta_{k}^{i}\right) A_{i} e_{k}^{i}+\sum_{j \in S_{n}^{i}}\left(1-\delta_{k}^{j}\right) A_{i j} e_{k}^{j}+w_{k}^{i} .
$$

Expression (7) suggests that stability of the error state $e_{k}^{i}$ for $i \in\{1, \ldots, N\}$ is sufficient to show the overall stability of the interconnected NCS. In consequence our focus is on investigating the stability of the error state $e_{k}^{i}$.

We propose a novel scheduling law which dynamically prioritizes the limited communication resources for an interconnected NCS. Assume the communication channel has the capacity constraint $c<N$, i.e. not all nodes can transmit at the same time. The following error-dependent scheduling rule defines the probability of channel access for each node $i$ at a time-step $k+1$ given the error values $e_{k}^{j}$ and error thresholds $\lambda_{j}$ for all $j \in\{1, \ldots, N\}$ at time-step $k$ by

$$
\mathrm{P}\left[\delta_{k+1}^{i}=1 \mid e_{k}^{j}, \lambda_{j}\right]= \begin{cases}0 & \left\|e_{k}^{i}\right\|_{Q_{i}}^{2} \leq \lambda_{i} \\ 1 & \left\|e_{k}^{i}\right\|_{Q_{i}}^{2}>\lambda_{i} \wedge n_{\lambda} \leq c \\ \frac{\left\|e_{k}^{i}\right\|_{Q_{i}}^{2}}{\sum_{n_{\lambda}}\left\|e_{k}^{j}\right\|_{Q_{i}}^{2}} & \left\|e_{k}^{i}\right\|_{Q_{i}}^{2}>\lambda_{i} \wedge n_{\lambda}>c\end{cases}
$$

where $\lambda_{i}$ is local error threshold for node $i$. The number of nodes satisfying $\left\|e_{k}^{i}\right\|_{Q_{i}}^{2}>\lambda_{i}$ is denoted $n_{\lambda}$, where we define $\left\|e_{k}^{i}\right\|_{Q_{i}}^{2}:=e_{k}^{i \top} Q_{i} e_{k}^{i}$ and $Q_{i}$ is a positive definite weight matrix. First, if $\left\|e_{k}^{i}\right\|_{Q_{i}}^{2} \leq \lambda_{i}$, the corresponding node is deterministically excluded from channel access competition. In case $n_{\lambda}>c$, the $c$ nodes are randomly selected such that the ones with larger errors have more chances of being selected, as indicated in the last line of (8). We assume that the scheduler is updated with error norms $\left\|e_{k}^{i}\right\|_{Q_{i}}^{2} \in \mathbb{R}$ from all nodes $i$ at every time $k$. This can be realized by considering a low-capacity channel between the scheduler and sub-systems to send those $N$ real numbers to the scheduler. The randomization in the last line of (8) allows to implement it approximately in decentralized fashion [13].
We define the aggregate error state $e_{k} \in \mathbb{R}^{n}$ by stacking the error vectors of all sub-systems $i \in\{1, \ldots, N\}$ as follows:

$$
e_{k}=\left[e_{k}^{1 \top}, \ldots, e_{k}^{N \top}\right]^{\top},
$$

The scheduling policy in (8) is a randomized policy depending only on the most recent error values. Since our policy is forgetful about the error values $e_{m}, m<k$, (9) defines a random process (7) possessing Markov property. The Markov chain $e_{k}$ is homogeneous since the difference equation (4) is time-invariant and the noise process $w_{k}^{i}$ is i.i.d. for all $i \in\{1, \ldots, N\}$. Moreover, the distribution $w_{k}^{i}$ is absolutely continuous with positive density function at any state $e_{k}$ implying the chain is aperiodic and $\psi$-irreducible.

For ease of presentation we impose the capacity constraint such that one node can only transmit at each time-step $k$ :

$$
\sum_{i=1}^{N} \delta_{k}^{i}=c=1
$$

The presented results readily extend towards $c>1$.

\section{A. Preliminaries}

A common stability notion for uncountable Markov chains is given by $f$-ergodicity, defined in the following:

Definition 1: [15] Let $f: \mathbb{R}^{n} \rightarrow[1,+\infty)$ be a real-valued function. Then, a Markov chain $\Phi$ is called $f$-ergodic, if

1) $\Phi$ is positive Harris recurrent with the unique invariant probability measure $\pi$,

2) the expectation $\pi(f):=\int f\left(\Phi_{k}\right) \pi\left(d \Phi_{k}\right)$ is finite. Thus, ergodicity confirms the existence of an invariant finite distribution over the entire state-space, implying that the process returns to some petite sets in finite time.

Definition 2: Let $V: \mathbb{R}^{n} \rightarrow \mathbb{R}^{+}$be a real-valued measurable function of Markov chain $\Phi$. The drift operator $\Delta$ is

$$
\Delta V\left(\Phi_{k}\right)=\mathrm{E}\left[V\left(\Phi_{k+1}\right) \mid \Phi_{k}\right]-V\left(\Phi_{k}\right), \quad \Phi_{k} \in \mathbb{R}^{n} .
$$

Theorem 1 ( $f$-Norm Ergodic Theorem [15]): Suppose that Markov chain $\Phi$ is $\psi$-irreducible and aperiodic and let $f \geq 1$ be a real-valued function in $\mathbb{R}^{n}$. If a small set $\mathcal{D}$ and a non-negative real-valued function $V$ satisfying $V\left(\Phi_{0}\right)<\infty$ exists such that $\Delta V(\Phi) \leq-f(\Phi)$ for any $\Phi \in \mathbb{R}^{n} \backslash \mathcal{D}$ and $\Delta V<\infty$ for $\Phi \in \mathcal{D}$, then $\Phi$ is $f$-ergodic.

Remark 2: All compact subsets of a linear state-space are small sets [15, Sec. 5.3.5]. It is in addition well-known that all small sets are petite [15, Sec. 5.5.2].

\section{StABILITY ANALYSIS}

In this section, we study the behavior of interconnected NCSs, under the scheduling policy (8). Having the concatenated system (2) stabilized in the absence of the communication constraint, the triangularity of (6) allows us to invoke Theorem 1 to solely analyze the error state $e_{k}$. First, define a non-negative real-valued function $V\left(e_{k}\right): \mathbb{R}^{n} \rightarrow \mathbb{R}^{+}$as

$$
V\left(e_{k}\right)=\sum_{i=1}^{N} e_{k}^{i^{\top}} Q_{i} e_{k}^{i}=\sum_{i=1}^{N}\left\|e_{k}^{i}\right\|_{Q_{i}}^{2} .
$$

Due to characteristics of the selected function (12), employing the drift $\Delta V$ over one transition step, i.e. $k \rightarrow k+1$ for the Markov chain (9) becomes too conservative. We illustrate this observation by the following example: 
Consider two identical scalar systems competing for one channel slot at each time, with $A_{1}=A_{2}=A, A_{12} \neq 0$ while $A_{21}=0$. For illustration purposes, assume $Q_{1}=Q_{2}=1$ and $e_{k}^{1}=e_{k}^{2}=\bar{e}_{k}>\lambda_{1}=\lambda_{2}$. Thus, the transmission chance for each node at time $k+1$ is $\frac{1}{2}$ according to (8). From (7) and (12), the one-step drift (11) can be calculated as

$$
\begin{aligned}
\Delta V\left(e_{k}\right) & =\mathrm{E}\left[V\left(e_{k+1}\right) \mid e_{k}\right]-V\left(e_{k}\right) \\
& =2+\left\|A \overline{e_{k}}\right\|_{2}^{2}+\frac{1}{2}\left\|A_{12} \overline{e_{k}}\right\|_{2}^{2}-2\left\|\overline{e_{k}}\right\|_{2}^{2} .
\end{aligned}
$$

For $A>\sqrt{2}$ or $A_{12}>2$, the drift is positive, violating Theorem 1. Due to the constraint (10), some sub-systems are obliged to operate in open-loop between their two consecutive transmissions. Hence, their respective local errors are expected to grow. Thus, looking at one-step transition of the Markov chain might be too conservative to show the ergodicity. Considering an interval with length $N$ ensures that enough transmission possibilities are provided, although it does not guarantee that a sub-system certainly transmits over the interval. Remember that ergodicity over an interval implies ergodicity over longer intervals [15]. To infer $f$ ergodicity over the interval $[k, k+N]$, we modify (11) as

$$
\Delta V\left(e_{k}, N\right)=\mathrm{E}\left[V\left(e_{k+N}\right) \mid e_{k}\right]-V\left(e_{k}\right), e_{k} \in \mathbb{R}^{n} .
$$

It is now essential to observe how the error of a node $i$ evolves over an interval. Employing (7), $e_{k+N}^{i}$ is written as a function of the error at an arbitrary time $k+r_{i} \in[k, k+N]$ :

$$
\begin{aligned}
& e_{k+N}^{i}=\prod_{\alpha=r_{i}+1}^{N}\left(1-\delta_{k+\alpha}^{i}\right) A_{i}^{N-r_{i}} e_{k+r_{i}}^{i} \\
& +\underbrace{\sum_{r=r_{i}}^{N-1}\left[\prod_{\alpha=r+2}^{N}\left(1-\delta_{k+\alpha}^{i}\right) A_{i}^{N-r-1} w_{k+r}^{i}\right]}_{\text {Corresponds to local dynamics of node } i} \\
& +\sum_{j \in S_{n}^{i}}\left[\sum_{\beta=0}^{N-r_{i}-1} \prod_{\gamma=r_{i}+1}^{r_{i}+\beta+1}\left(1-\delta_{k+\gamma}^{j}\right) \prod_{\kappa=r_{i}+\beta+2}^{N}\left(1-\delta_{k+\kappa}^{i}\right) A_{i}^{\bar{\beta}_{1}} A_{i j} A_{j}^{\beta}\right] e_{k+r_{i}}^{j} \\
& +\sum_{j \in S_{n}^{i}}\left[\sum_{\beta=0}^{N-r_{i}-2} \prod_{\gamma=r_{i}+2}^{r_{i}+\beta+2}\left(1-\delta_{k+\gamma}^{j}\right) \prod_{\kappa=r_{i}+\beta+3}^{N}\left(1-\delta_{k+\kappa}^{i}\right) A_{i}^{\bar{\beta}_{2}} A_{i j} A_{j}^{\beta}\right] w_{k+r_{i}}^{j} \\
& +\ldots \\
& +\sum_{j \in S_{n}^{i}}\left[\sum_{\beta=0}^{1} \prod_{\gamma=N-1}^{N+\beta-1}\left(1-\delta_{k+\gamma}^{j}\right) \prod_{\kappa=N+\beta}^{N}\left(1-\delta_{k+\kappa}^{i}\right) A_{i}^{1-\beta} A_{i j} A_{j}^{\beta}\right] w_{k+N-3}^{j} \\
& +\underbrace{\sum_{j \in S_{n}^{i}}\left(1-\delta_{k+N}^{j}\right) A_{i j} w_{k+N-2}^{j}}_{\text {The effect of direct neighboring nodes } j \in S_{n}^{i}} \\
& +\sum_{j \in S_{n}^{i}} \sum_{l \in S_{n}^{j}} \mathcal{F}_{e}\left(\delta^{i}, \delta^{j}, \delta^{l}, A_{i}, A_{j}, A_{l}, A_{i j}, A_{j l}\right) e_{k+r_{i}}^{l} \\
& +\sum_{j \in S_{n}^{i}} \sum_{l \in S_{n}^{j}} \mathcal{F}_{w}\left(\delta^{i}, \delta^{j}, \delta^{l}, A_{i}, A_{j}, A_{l}, A_{i j}, A_{j l}\right) w_{\left[k+r_{i}: k+N-3\right]}^{l} \\
& +\ldots \\
& +\underbrace{\sum_{j \in S_{n}^{i}} \sum_{l \in S_{n}^{j}} \ldots \sum_{o \in S_{n}^{t}}\left(1-\delta_{k+N}^{j}\right)\left(1-\delta_{k+N-1}^{l}\right) \ldots\left(1-\delta_{k+r_{i}+1}^{o}\right) \bar{A}_{i o} e_{k+r_{i}}^{o}}_{\text {The effect of multi-hop neighbors of node } i}
\end{aligned}
$$

where, $\bar{\beta}_{1}=N-r_{i}-\beta-1, \bar{\beta}_{2}=N-r_{i}-\beta-2, \bar{A}_{i o}=$ $A_{i j} A_{j l} \ldots A_{t o}$. The two matrices $\mathcal{F}_{e}$ and $\mathcal{F}_{w}$ are not given explicitly due to space limitations, but they represent the effect of two-hop neighbors $l \in S_{n}^{j}$. The expression (14) is extremely complex to be analyzed due to its distributed nature. Indeed to analyze the behavior of a single node $i$, the behavior of all nodes need to be observed at all timesteps. Thus, we confine our focus on a special class of interconnected NCSs where the interconnections are modeled by DAGs. Fig. 2 illustrates a sample DAG with eight nodes.
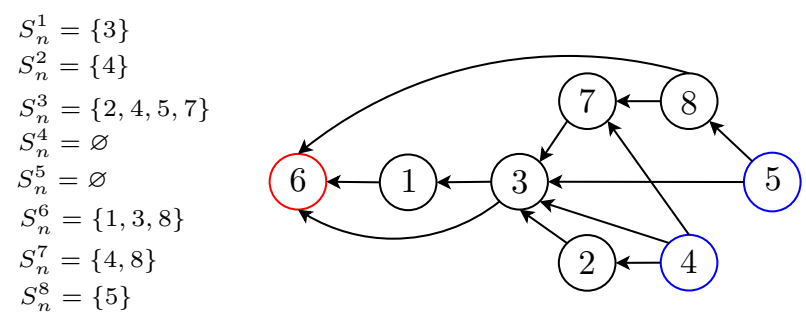

Fig. 2. A DAG with eight nodes and twelve directed edges. Node 6 is the only-affected node while nodes 4 and 5 are the only-affecting nodes.

Two main properties of DAGs are: 1) There exists no cycle which ensures the Markov property, 2) If node $j$ is either a direct or multi-hop neighbor of node $i$, i.e. $j \in \tilde{S}_{n}^{i}$, then node $i$ is not a direct nor a multi-hop neighbor of node $j$, i.e. $i \notin \tilde{S}_{n}^{j}$. These two properties play essential roles in analyzing stability of the Markov chain (9). Having DAGs, we are always able to divide the nodes into different layers, from the layer including only-affecting nodes to the layer containing only-affected nodes. The only-affecting nodes possess only local dynamics as they are not affected (see nodes 4 and 5 in Fig. 2). Thus, we analyze (14) by initially looking at only-affecting nodes. In fact, given an only-affecting node $j$ which admits an $f$-ergodic error state, then $f$-ergodicity of an affected node $i$ is preserved if $j$ is a neighbor of $i$. In Fig. 2, nodes 4 and 5 are only-affecting. Showing stability of these nodes, then nodes 2 and 8 are also stable only if their respective local dynamics (excluding the effect from nodes 4 and 5) are stable. The stability of nodes 4 and 8 ensure the stability of node 7 , if the local dynamics of node 7 is stable. We can continue this scenario until the only-affected node 6 . Following this approach enables us to look at the error state of each node independent of the neighboring effects.

Theorem 2: Consider an interconnected NCS with $N$ heterogeneous LTI sub-systems given in (1) and a communication channel subject to the constraint (10), and the control, estimation and scheduling laws given by (3), (4) and (8), respectively. Let the interconnections between sub-systems be modeled by a DAG $\mathcal{G}_{c}(\mathcal{V}, \mathcal{E})$. Then appropriate $\lambda_{i}$ 's and $Q_{i}$ 's exist such that the Markov chain (9) is $f$-ergodic.

Proof: To invoke Theorem 1 for the local error state $e_{k}^{i}$, we need to check if they possess the Markov property under the scheduling law (8). Considering a DAG as the interconnection model, it follows that for a specific node $i$ at a time-step $k+r_{i}$, the scheduling law (8) contains error values only from one time-step earlier, i.e. $e_{k+r_{i}-1}^{i}$. This conclusion follows from acyclic property of DAGs. Even if node $i$ is a neighbor of a node $j$, this effect appears via the term $A_{j i} e_{k+r_{i}-1}^{i}$ which does not violate the Markov property. The homogeneity of (7) follows since (4) is time-invariant and $w_{k}^{i}$ 
is i.i.d. for $i=\{1, \ldots, N\}$. Furthermore, having continuous noise distributions $w_{k}^{i}$ with positive density functions implies that (7) is an aperiodic and $\psi$-irreducible Markov chain.

We study stability of each sub-system considering only its local dynamics, i.e. we look at the first two terms of (14):

$$
\begin{aligned}
e_{k+N}^{i, \text { local }} & =\prod_{\alpha=r_{i}+1}^{N}\left(1-\delta_{k+\alpha}^{i}\right) A_{i}^{N-r_{i}} e_{k+r_{i}}^{i} \\
& +\sum_{r=r_{i}}^{N-1}\left[\prod_{\alpha=r+2}^{N}\left(1-\delta_{k+\alpha}^{i}\right) A_{i}^{N-r-1} w_{k+r}^{i}\right] .
\end{aligned}
$$

We assume that each sub-system has operated from timestep $k$ to $k+N-1$ utilizing the policy (8). Then, the last time $k+N$ is scheduled considering all possible scenarios over $[k, k+N-1]$. It should be noted that the scheduling unit receives true error values from each node and decides the priorities considering the interconnections as well. Therefore, the error values in (8) are given by the expression (14). We define at every time $k^{\prime} \in[k, k+N]$ two time-varying disjoint sets $S_{k^{\prime}}^{1}$ and $S_{k^{\prime}}^{2}$, such that for every node $i \in\{1, \ldots, N\}$

$$
i \in\left\{\begin{array}{ll}
S_{k^{\prime}}^{1} & \text { if }\left\|e_{k^{\prime}}^{i}\right\|_{Q_{i}}^{2} \leq \lambda_{i} \\
S_{k^{\prime}}^{2} & \text { if }\left\|e_{k^{\prime}}^{i}\right\|_{Q_{i}}^{2}>\lambda_{i}
\end{array},\right.
$$

with $S_{k^{\prime}}^{1} \cup S_{k^{\prime}}^{2}=N$. Eligible nodes to transmit at time $k^{\prime}+1$ are included in $S_{k^{\prime}}^{2}$, while $S_{k^{\prime}}^{1}$ contains the excluded nodes from access competition. Note that, transmission eligibility of a node depends on both transmission occurrence and noise process. To take this into account we discern three complementary and disjoint cases for a node $i$ as follows:

$c_{1}$ : Node $i$ has either transmitted or not within the past $N-1$ time-steps, and is in set $S_{k+N-1}^{1}$, i.e.

$$
i \in S_{k+N-1}^{1} \quad \Rightarrow \quad\left\|e_{k+N-1}^{i}\right\|_{Q_{i}}^{2} \leq \lambda_{i}
$$

$c_{2}$ : Node $i$ has transmitted at least once within the past $N-1$ time-steps, and is in set $S_{k+N-1}^{2}$, i.e.

$\exists k^{\prime} \in[k, k+N-1]: \delta_{k^{\prime}}^{i}=1$ and $\left\|e_{k+N-1}^{i}\right\|_{Q_{i}}^{2}>\lambda_{i}$,

$c_{3}$ : Node $i$ has not transmitted within the past $N-1$ timesteps, and is in set $S_{k+N-1}^{2}$, i.e.

$\forall k^{\prime} \in[k, k+N-1]: \delta_{k^{\prime}}^{i}=0$ and $\left\|e_{k+N-1}^{i}\right\|_{Q_{i}}^{2}>\lambda_{i}$.

Each node is characterized by only one of the above cases during transition from $k$ to $k+N-1$. We apply Theorem 1 to the cases $c_{1}-c_{3}$ employing the drift (13) and Lyapunov function (12). Remember that we consider local error of each node. The $N$-step drift (13) is split into partial drifts as

$$
\Delta V\left(e_{k}^{i \in c_{j}}, N\right)=\sum_{c_{j}} \mathrm{E}\left[\left\|e_{k+N}^{i, \text { local }}\right\|_{Q_{i}}^{2} \mid e_{k}\right]-V\left(e_{k}^{c_{j}}\right),
$$

where $V\left(e_{k}^{c_{j}}\right)=\sum_{i \in c_{j}}\left\|e_{k}^{i}\right\|_{Q_{i}}^{2}$, for $\forall i \in c_{j}$ and $j \in\{1,2,3\}$.

For $i \in c_{1}$ it follows $\left\|e_{k+N-1}^{i}\right\|_{Q_{i}}^{2} \leq \lambda_{i}$. This implies $\delta_{k+N}^{i}=0$ for $\forall i \in c_{1}$. Consider $r_{i}=N-1$ in (15), then

$$
\begin{aligned}
& \sum_{c_{1}} \mathrm{E}\left[\left\|e_{k+N}^{i, \text { local }}\right\|_{Q_{i}}^{2} \mid e_{k}\right]=\sum_{c_{1}} \mathrm{E}\left[\left\|A_{i} e_{k+N-1}^{i}+w_{k+N-1}^{i}\right\|_{Q_{i}}^{2} \mid e_{k}\right] \\
& \leq \sum_{c_{1}}\left\|A_{i}\right\|_{2}^{2} \mathrm{E}\left[\left\|e_{k+N-1}^{i}\right\|_{Q_{i}}^{2} \mid e_{k}\right]+\mathrm{E}\left[\left\|w_{k+N-1}^{i}\right\|_{Q_{i}}^{2}\right] \\
& \leq \sum_{c_{1}}\left\|A_{i}\right\|_{2}^{2} \lambda_{i}+\operatorname{tr}\left(Q_{i}\right) .
\end{aligned}
$$

According to (17), the partial drift then becomes

$$
\Delta V\left(e_{k}^{c_{1}}, N\right) \leq \sum_{c_{1}}\left\|A_{i}\right\|_{2}^{2} \lambda_{i}+\operatorname{tr}\left(Q_{i}\right)-V\left(e_{k}^{c_{1}}\right) .
$$

With $f_{c_{1}}:=\epsilon_{1} V\left(e_{k}^{c_{1}}\right)-\xi_{1}^{b^{+}}$, where $\xi_{1}^{b^{+}}$stands for the upper bound (18) and $\epsilon_{1} \in(0,1]$. We can then find a small set $\mathcal{D}_{1}$ and constant $\epsilon_{1}$ such that $f_{c_{1}} \geq 1$ and $\Delta V\left(e_{k}^{c_{1}}, N\right) \leq-f_{c_{1}}$.

For a node $i \in c_{2}$, assume that the last transmission occurred at time $k+r_{i}$, i.e. $\delta_{k+r_{i}}^{i}=1$. Statistical independence of the sequence $w_{k+r}^{i}$ and error $e_{k+r_{i}-1}^{i}$ follows

$$
\begin{gathered}
\sum_{c_{2}} \mathrm{E}\left[\left\|e_{k+N}^{i, \text { local }}\right\|_{Q_{i}}^{2} \mid e_{k}\right]=\sum_{c_{2}} \sum_{r=r_{i}}^{N-1} \mathrm{E}\left[\left\|A_{i}^{N-r-1} w_{k+r}^{i}\right\|_{Q_{i}}^{2}\right] \\
\leq \sum_{c_{2}} \sum_{r=r_{i}}^{N-1}\left\|A_{i}^{N-r-1}\right\|_{2}^{2} \operatorname{tr}\left(Q_{i}\right)
\end{gathered}
$$

From (17), it follows

$$
\Delta V\left(e_{k}^{c_{2}}, N\right) \leq \sum_{c_{2}} \sum_{r=r_{i}}^{N-1}\left\|A_{i}^{N-r-1}\right\|_{2}^{2} \operatorname{tr}\left(Q_{i}\right)-V\left(e_{k}^{c_{2}}\right) .
$$

Define $f_{c_{2}}=\epsilon_{2} V\left(e_{k}^{c_{2}}\right)-\xi_{2}^{b^{+}}$, where $\xi_{2}^{b^{+}}$is given in (19) and $\epsilon_{2} \in(0,1]$. Thus, we can find a small set $\mathcal{D}_{2} \subset \mathbb{R}^{n}$ and constant $\epsilon_{2}$ such that $f_{c_{2}} \geq 1$ and $\Delta V\left(e_{k}^{c_{2}}, N\right) \leq-f_{c_{2}}$.

The nodes in $c_{3}$ are eligible for channel access at final time $k+N$ as $i \in S_{k+N-1}^{2}$. To infer $f$-ergodicity, we split the case $c_{3}$ into two complementary and disjoint sub-cases:

$l_{1}^{c_{3}}$ node $i$ has not transmitted within the past $N-1$ timesteps, but has been in the set $S^{1}$ at least once,

$l_{2}^{c_{3}}$ node $i$ has not transmitted within the past $N-1$ timesteps, and has been in $S_{\bar{k}}^{2}$ for all $\bar{k} \in[k, k+N-1]$.

In sub-case $l_{1}^{c_{3}}$, suppose that $k+r_{i} \in[k, k+N-2]$ was the last time for which $i \in S_{k+r_{i}}^{1}$, which implies $\left\|e_{k+r_{i}}^{i}\right\|_{Q_{i}}^{2} \leq \lambda_{i}$. Knowing that $\delta_{\bar{k}}^{i}=0$ for $k \in[k, k+N-1]$, we reach

$$
\begin{aligned}
& \sum_{l_{1}^{c_{3}}} \mathrm{E}\left[\left\|e_{k+N}^{i, \text { local }}\right\|_{Q_{i}}^{2} \mid e_{k}\right] \leq \\
& \sum_{l_{1}^{c_{3}}}\left[\left\|A_{i}^{N-r_{i}}\right\|_{2}^{2} \lambda_{i}+\sum_{r=r_{i}}^{N-1}\left\|A_{i}^{N-r-1}\right\|_{2}^{2} \operatorname{tr}\left(Q_{i}\right)\right] .
\end{aligned}
$$

Define $f_{l_{1}^{c_{3}}}=\epsilon_{l_{1}^{c_{3}}} V\left(e_{k}^{l_{1}^{c_{3}}}\right)-\xi_{l_{1}^{c_{3}}}^{b^{+}}$, where $\xi_{l_{1}^{c_{3}}}^{b^{+}}$is given in (20), with $\epsilon_{l_{1}^{c_{3}}} \in(0,1]$. Thus, we can find small set $\mathcal{D}_{l_{1}^{c_{3}}}$ and $\epsilon_{l_{1}^{c_{3}}}$ such that $f_{l_{1}^{c_{3}}} \geq 1$, and $\Delta V\left(e_{k}^{l_{1}^{c_{3}}}, N\right) \leq-f_{l_{1}^{c_{3}}}$.

In sub-case $l_{2}^{c_{3}}$, we know $\left\|e_{\bar{k}}^{i}\right\|_{Q_{i}}^{2}>\lambda_{i}$ for all time-steps $\bar{k} \in[k, k+N-1]$. Considering (15) with $r_{i}=0$, we have

$$
\begin{aligned}
& \sum_{l_{2}^{c_{3}}} \mathrm{E}\left[\left\|e_{k+N}^{i, \text { local }}\right\|_{Q_{i}}^{2} \mid e_{k}\right] \\
& \leq \sum_{l_{2}^{c_{3}}} \mathrm{E}\left[\left\|A_{i}^{N} e_{k}^{i}+\sum_{r=0}^{N-1}\left[A_{i}^{N-r-1} w_{k+r}^{i}\right]\right\|_{Q_{i}}^{2} \mid e_{k}\right] \\
& \leq \sum_{l_{2}^{c_{3}}}\left\|A_{i}^{N}\right\|_{2}^{2} V\left(e_{k}^{l_{2}^{c_{3}}}\right)+\sum_{r=0}^{N-1}\left\|A_{i}^{N-r-1}\right\|_{2}^{2} \operatorname{tr}\left(Q_{i}\right)
\end{aligned}
$$

Expression (21) depends on $e_{k}^{i}$ via the term $V\left(e_{k}^{l_{2}^{c_{3}}}\right)$, thus it is not bounded for arbitrary initial values. As the considered cases cannot happen all together, we calculate the probability that sub-case $l_{2}^{c_{3}}$ happens under the scheduling law (8). Recall that the length of the interval equals $N$. Thus, if a node, say $i$, does not transmit for all time-steps $\bar{k} \in[k, k+N]$, then inevitably another node, say $j$, transmits more than 
once. Let $k+r_{j}$ denote the latest time node $j$ transmitted, i.e. $\delta_{k+r_{j}}^{j}=1$. Thus, the probability that $j \in c_{2}$ transmits again at final time $k+N$, in the presence of node $i \in l_{2}^{c_{3}}$ is

$$
\begin{aligned}
& \mathrm{P}\left[\delta_{k+N}^{j}=1 \mid \delta_{k+r_{j}}^{j}=1, \delta_{\bar{k}}^{i}=0,\left\|e_{\bar{k}}^{i}\right\|_{Q_{i}}^{2}>\lambda_{i}\right] \\
& =\mathrm{E}\left[\mathrm{P}\left[\delta_{k+N}^{j}=1 \mid e_{k}\right] \mid \delta_{k+r_{j}}^{j}=1, \delta_{\bar{k}}^{i}=0,\left\|e_{\bar{k}}^{i}\right\|_{Q_{i}}^{2}>\lambda_{i}\right] \\
& =\mathrm{E}\left[\frac{\left\|e_{k+N-1}^{j}\right\|_{Q_{j}}^{2}}{\sum_{i \in S^{2}}\left\|e_{k+N-1}^{i}\right\|_{Q_{i}}^{2}} \mid \delta_{k+r_{j}}^{j}=1, \delta_{\bar{k}}^{i}=0,\left\|e_{\bar{k}}^{i}\right\|_{Q_{i}}^{2}>\lambda_{i}\right] .
\end{aligned}
$$

So far, $f$-ergodicity of the error states for the cases $c_{1}, c_{2}$, and $l_{1}^{c_{3}}$ is shown without calculating the probability of occurrence of those cases. In sub-case $l_{2}^{c_{3}}$ however, this is not true without incorporating the probability of occurrence. Remember that the scheduler assigns the priorities based on true error values (14) and not the local values (15). Noting that for nodes $q \in c_{1}$, we have $\delta_{k+N}^{q}=0$, we reach

$$
\begin{aligned}
& \mathrm{P}_{l_{2}^{c_{3}}}=\mathrm{P}\left[\delta_{k+N}^{j}=1 \mid \delta_{k+r_{j}}^{j}=1, \delta_{\bar{k}}^{i}=0,\left\|e_{\bar{k}}^{i}\right\|_{Q_{i}}^{2}>\lambda_{i}\right] \\
& =\mathrm{E}\left[\frac{\left\|e_{k+N-1}^{j}\right\|_{Q_{j}}^{2}}{\left\|e_{k+N-1}^{j}\right\|_{Q_{j}}^{2}+\sum_{i \in\left\{c_{2}, c_{3}\right\}}^{i \neq j}\left\|e_{k+N-1}^{i}\right\|_{Q_{i}}^{2}} \mid z_{i, j}\right] \\
& \leq \mathrm{E}\left[\frac{\left\|e_{k+N-1}^{j}\right\|_{Q_{j}}^{2}}{\left\|e_{k+N-1}^{j}\right\|_{Q_{j}}^{2}+\sum_{i \in c_{2}}^{i \neq j} \lambda_{i}+\sum_{i \in l_{1}^{c_{3}}} \lambda_{i}+\sum_{i \in l_{2}^{c_{3}} \lambda_{i}}} \mid z_{i, j}\right],
\end{aligned}
$$

where $z_{i, j}$ abbreviates the conditions of the expectation. Due to the linearity of sub-systems and the error dynamics (7) and since $\left\|e_{k}^{i}\right\|_{Q_{i}}<\infty$, we conclude $\left\|e_{k+N-1}^{j}\right\|_{Q_{j}}^{2}<\infty$. Thus, by selecting appropriate $\lambda_{i}$ 's and $Q_{i}$ 's we can make the latest inequality arbitrarily close to zero. Intuitively, the probability of subsequent transmissions for a certain node, in presence of nodes with large errors and without prior transmissions, can be made arbitrarily close to zero by tuning $\lambda_{i}$ 's and $Q_{i}$ 's. In fact, by increasing $\lambda_{j}$ for $j \notin l_{2}^{c_{3}}$, more of them are left out of channel competition in favor of nodes in $l_{2}^{c_{3}}$. The $N$-step drift defined in (13) can be expressed in terms of partial drifts considering the probabilities of happening different cases as

$$
\Delta V\left(e_{k}, N\right)=\sum_{c_{j}} \mathrm{P}_{c_{j}} \mathrm{E}\left[\left\|e_{k+N}^{i, \text { local }}\right\|_{Q_{i}}^{2}\right]-V\left(e_{k}^{c_{j}}\right),
$$

where, $\mathrm{P}_{c_{j}}$ is the occurrence probability of cases $c_{j}$, such that $\sum_{c_{j}} \mathrm{P}_{c_{j}}=1$. Therefore, the overall drift can be expressed as

$$
\begin{aligned}
\Delta V\left(e_{k}, N\right) & \leq \Delta V\left(e_{k}^{c_{1}}, N\right)+\Delta V\left(e_{k}^{c_{2}}, N\right)+\Delta V\left(e_{k}^{l_{1}^{c_{3}}}, N\right) \\
& +\sum_{l_{2}^{c_{3}}} \mathrm{P}_{l_{2}^{c_{3}}} \mathrm{E}\left[\left\|e_{k+N}^{i, \text { local }}\right\|_{Q_{i}}^{2}\right]-V\left(e_{k}^{l_{2}^{c_{3}}}\right) \\
& \leq-\left[f_{c_{1}}+f_{c_{2}}+f_{l_{1}^{c_{3}}}+f_{l_{2}^{c_{3}}}\right]=-f\left(e_{k}\right),
\end{aligned}
$$

where, in the upperbound $\mathrm{P}_{c_{1}}=\mathrm{P}_{c_{2}}=\mathrm{P}_{l_{1}^{c_{3}}}=1$ is assumed .

Thus for the sub-case $l_{2}^{c_{3}}$, and with (21) we have

$$
\begin{aligned}
\mathrm{P}_{l_{2}^{c_{3}}} & {\left[\sum_{l_{2}^{c_{3}}} \mathrm{E}\left[\left\|e_{k+N}^{i, \text { local }}\right\|_{Q_{i}}^{2} \mid e_{k}\right]\right]-V\left(e_{k}^{l_{2}^{c_{3}}}\right) } \\
\leq & {\left[\mathrm{P}_{l_{2}^{c_{3}}} \sum_{l_{2}^{c_{3}}}\left\|A_{i}^{N}\right\|_{2}^{2}-1\right] V\left(e_{k}^{l_{2}^{c_{3}}}\right)+\xi_{l_{2}^{c_{3}}}^{b^{+}}, }
\end{aligned}
$$

where $\xi_{l_{2}^{c_{3}}}^{b^{+}}=\mathrm{P}_{l_{2}^{c_{3}}} \sum_{l_{2}^{c_{3}}} \sum_{r=0}^{N-1}\left\|A_{i}^{N-r-1}\right\|_{2}^{2} \operatorname{tr}\left(Q_{i}\right)$. Define $f_{l_{2}^{c_{3}}}=\epsilon_{l_{2}^{c_{3}}} V\left(e_{k}^{l_{2}^{c_{3}}}\right)-\xi_{l_{2}^{c_{3}}}^{b^{+}}$, with $\epsilon_{l_{2}^{c_{3}}} \in(0,1]$. We can

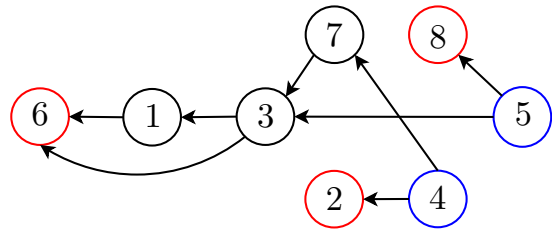

Fig. 3. The DAG of interconnections between sub-systems in an NCS.

find appropriate $\lambda_{i}$ 's and $Q_{i}$ 's then such that small set $\mathcal{D}_{l_{2}^{c_{3}}}$ and $\epsilon_{l_{2}^{c_{3}}}$ exist satisfying $f_{l_{2}^{c_{3}}} \geq 1$, and $\Delta V\left(e_{k}^{l_{2}^{c_{3}}}, N\right) \leq-f_{l_{2}^{c_{3}}}$.

We have shown the conditions of Theorem 1 hold separately for each case $c_{j}$. As the cases are complementary and disjoint, we define the small set $\mathcal{D}_{f} \subset \mathbb{R}^{n}$ and $\epsilon_{f} \in(0,1]$ such that $f\left(e_{k}\right) \geq 1$, and $\Delta V\left(e_{k}, N\right) \leq-f\left(e_{k}\right)$. This confirms that Theorem 1 holds for the overall drift (22), which proves the Markov chain (15) is $f$-ergodic. This yields that the random error values for each sub-system are selected from bounded variance distributions. This proves the $f$-ergodicity of local error state (15) and consequently the $f$-ergodicity of (14).

Remark 3: For general interconnection structures with undirected edges and cycles, the ergodicity of the error (14) cannot be guaranteed. This follows from the fact that the error of a node $i$ at some prior time-steps might appear in its dynamics again in future through the neighboring nodes, which violates the Markov property. However, the DAG assumption on the control structure can be relaxed, which in turn introduces extra coupling terms in the system state dynamics $x_{k}^{i}$, i.e. in the first row of the squared matrix in (6).

\section{NUMERICAL RESULTS}

To illustrate the performance of our scheduling design, consider an interconnected NCS comprised of eight scalar sub-systems competing for the sole channel slot at each timestep. The interconnection between sub-systems is depicted in Fig. 3, where the red and blue nodes represent only-affected and only-affecting nodes, respectively. Consider a class of identical sub-systems including nodes $\{3,4,5,6\}$, with stable systems and parameters $A_{1}=0.7, B_{1}=1$ and $\lambda_{1}=5$. The second class includes the remaining nodes $\{1,2,7,8\}$ having unstable systems with parameters $A_{2}=1.2, B_{2}=1$ and $\lambda_{2}=3$. Both classes start from zero initial condition and $w_{k}^{i} \sim \mathcal{N}(0,1)$. To stabilize local systems, we choose a deadbeat control law, i.e. $L_{i}=A_{i}$ and $L_{i j}=A_{i j}$ for all $i, j$ and a model-based observer given by (4). Next we select $Q_{i}=I$. For simplicity, assume all interconnection matrices $A_{i j}=A_{\text {int }}$ are identical. We derive the numerical results under the constraint (10) and the scheduling law (8).

Fig. 4 provides the average error variances for nodes $\{1, \ldots, 8\}$ for different interconnection strengths $A_{\text {int }}=$ $\{0.1,0.2,0.3,0.4,0.5\}$. The averages are calculated via Monte Carlo simulation over $5 \times 10^{4}$ samples. The lower bound is derived by ignoring the constraint (10), i.e. every sub-system transmits at every time-step.

The results indicate that the average error variances expectedly increase with increasing interconnection 


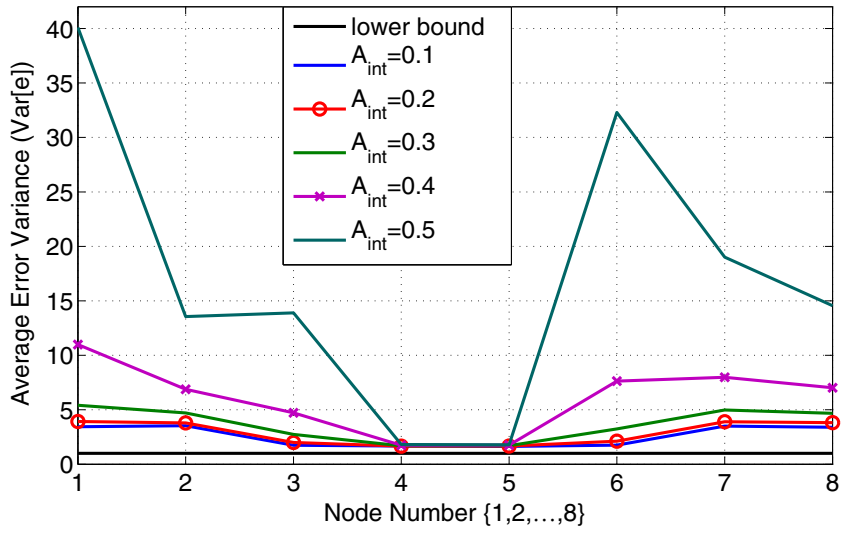

Fig. 4. Error variances for each node of the DAG in Fig. 3.

strength $A_{\text {int }}$. The error variances corresponding to nodes 4 and 5 change only slightly as those are only-affecting nodes and they possess no neighboring effect. The slight increase in their corresponding variances follows from the fact that by increasing $A_{\text {int }}$, transmission chances for nodes 4 and 5 decrease, as the access probabilities for the affected nodes increase. Thus, nodes 4 and 5 transmit less occasionally, which generally leads to an increase of their error values. The error variance corresponding to node 6 grows rapidly by increasing $A_{\text {int }}$, which results from being the most affected node. In addition, the higher growth rate of error variances for nodes $\{1,2,7,8\}$ follows from having unstable systems.

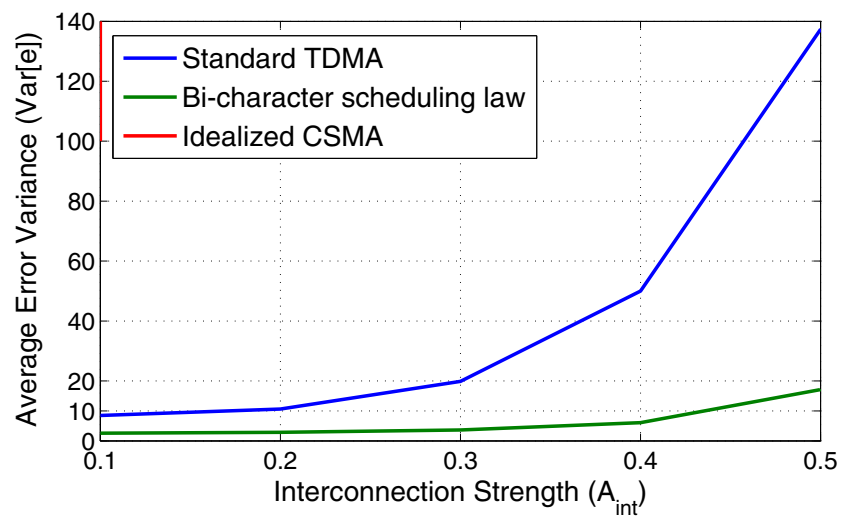

Fig. 5. Comparison of error variances for different scheduling protocols.

In Fig. 5 the performance of our proposed policy is compared with the standard TDMA and idealized CSMA protocols. The results are obtained for TDMA considering each node transmits exactly every eight time-steps. This is the simplest pattern one might choose for TDMA and does not take the system parameters into account. However, finding the optimal pattern for TDMA over infinite horizon is an open problem, while searching for patterns which outperform the standard TDMA is not straightforward, especially for interconnected systems. It can be seen that the event-based prioritized scheme outperforms the standard TDMA in terms of error variance. In addition, a comparison with the idealized CSMA protocol, which is a uniform random access scheme, is performed. Considering the uniform access probability $\frac{1}{8}$ for each node at each time-step, the average error variance for the case $A_{\text {int }}=0.1$ has the order of $10^{9}$. This is expected since employing idealized CSMA results in a probable nontransmission state for a node with rapidly growing error.

\section{CONCLUSIONS}

In this paper we propose an event-based error-dependent scheduling law for a class of resource-constrained interconnected NCSs. Within this class, the interconnections are modeled by directed acyclic graphs (DAGs). Given a stabilizing control law in the absence of communication constraints, we show $f$-ergodicity of the overall network-induced error for this class of interconnected NCSs. Simulation results are in accordance with stability results and show performance improvement in comparison with the time-triggered and pure randomized protocols such as TDMA and idealized CSMA.

\section{REFERENCES}

[1] D. D. Siljak, Decentralized Control of Complex Systems. Mathematics in Science and Engineering, Academic Press, 1991.

[2] C. Langbort, R. Chandra, and R. D'Andrea, "Distributed control design for systems interconnected over an arbitrary graph," IEEE Trans. on Automatic Cont., vol. 49, no. 9, pp. 1502-1519, 2004.

[3] F. Deroo, M. Meinel, M. Ulbrich, and S. Hirche, "Distributed control design with local model information and guaranteed stability," in 19th IFAC World Congress, 2014.

[4] D. Dimarogonas, E. Frazzoli, and K. Johansson, "Distributed eventtriggered control for multi-agent systems," Automatic Control, IEEE Transactions on, vol. 57, pp. $1291-1297$, may 2012.

[5] A. Molin and S. Hirche, "On the optimality of certainty equivalence for event-triggered control systems," Automatic Control, IEEE Transactions on, vol. 58, no. 2, pp. $470-474,2013$.

[6] G. C. Walsh, H. Ye, and L. G. Bushnell, "Stability analysis of networked control systems," Control Systems Technology, IEEE Transactions on, vol. 10, no. 3, pp. 438-446, 2002.

[7] D. Nesic and A. Teel, "Input-output stability properties of networked control systems," Automatic Control, IEEE Transactions on, vol. 49, no. 10 , pp. $1650-1667,2004$.

[8] D. Christmann, R. Gotzhein, S. Siegmund, and F. Wirth, "Realization of Try-Once-Discard in wireless multihop networks," IEEE Transactions on Industrial Informatics, vol. 10, pp. 17-26, Feb 2014.

[9] C. Ramesh, H. Sandberg, and K. Johansson, "Stability analysis of multiple state-based schedulers with CSMA," in Decision and Control (CDC), 2012 IEEE 51 st Annual Conference on, pp. 7205-7211, 2012.

[10] M. Mamduhi, A. Molin, and S. Hirche, "Event-based scheduling of multi-loop stochastic systems over shared communication channels," in 21st International Symposium on Mathematical Theory of Networks and Systems (MTNS), pp. 266-273, 2014.

[11] W. H. Heemels, A. R. Teel, N. van de Wouw, and D. Nesic, "Networked control systems with communication constraints: Tradeoffs between transmission intervals, delays and performance," Automatic Control, IEEE Transactions on, vol. 55, no. 8, pp. 1781-1796, 2010.

[12] M. Mamduhi, A. Molin, and S. Hirche, "Event-triggered scheduling for stochastic multi-loop networked control systems with packet dropouts," in Decision and Control (CDC), 2014 53rd IEEE Annual Conference on, pp. 2776-2782, Dec 2014.

[13] M. Mamduhi, D. Tolic, and S. Hirche, "Decentralized event-based scheduling for shared-resource networked control systems," in 14th Annual European Control Conference (ECC), pp. 941-947, Jul 2015.

[14] A. A. Alam, A. Gattami, and K. H. Johansson, "Suboptimal decentralized controller design for chain structures: Applications to vehicle formations," in 50th IEEE Conference on Decision and Control and European Control Conference (CDC-ECC), pp. 6894-6900, 2011.

[15] S. Meyn and R. Tweedie, Markov chains and stochastic stability. Springer London, 1996. 